\title{
Building Roof Plane Extraction From LIDAR Data
}

\author{
Mohammad Awrangjeb and Guojun Lu \\ Gippsland School of Information Technology, Churchill Vic 3842 Australia, Phone: +61 399026462 \\ E-mail: \{Mohammad.Awrangjeb, Guojun.Lu\}@ monash.edu, Web: http://users.monash.edu.au/ mawrangj/
}

\begin{abstract}
This paper presents a new segmentation technique to use LIDAR point cloud data for automatic extraction of building roof planes. The raw LIDAR points are first classified into two major groups: ground and non-ground points. The ground points are used to generate a 'building mask' in which the black areas represent the ground where there are no laser returns below a certain height. The non-ground points are segmented to extract the planar roof segments. First, the building mask is divided into small grid cells. The cells containing the black pixels are clustered such that each cluster represents an individual building or tree. Second, the non-ground points within a cluster are segmented based on their coplanarity and neighbourhood relations. Third, the planar segments are refined using a rulebased procedure that assigns the common points among the planar segments to the appropriate segments. Finally, another rule-based procedure is applied to remove tree planes which are generally small in size and randomly oriented. Experimental results on three Australian sites have shown that the proposed method offers high building detection and roof plane extraction rates.
\end{abstract}

\section{INTRODUCTION}

Automatic reconstruction of buildings from aerial imagery and/or LIDAR (Light Detection And Ranging) data is a prerequisite for many GIS (Geographic Information System) applications, such as 3D building modelling and building change detection. There are two types of building reconstruction techniques in the literature: semiautomatic and automatic. Since the semiautomatic techniques require high volume of human involvements to reconstruct buildings even in a small city, the obvious option is looking for the automatic systems.

Different approaches to building roof reconstruction have been reported in the literature [1]. In the model driven approach, also known as the parametric approach, a predefined catalogue of roof forms (eg, flat, saddle, etc) is prescribed, and the model that best fits the data is chosen. In the data driven approach, also known as the generic approach or polyhedral approach, the roof is reconstructed from planar patches derived from segmentation algorithms. The structural approach, which is also known as the global strategy or Hybrid approach, exhibits both model and data driven characteristics. A recent update on the current reconstruction techniques is in [2].

This paper presents an automatic data driven approach to the extraction of building roof planes from LIDAR point cloud data. The raw LIDAR points are divided into two groups using a height threshold. The first group contains the ground points that are exploited to generate a binary building mask where buildings and trees are found as black shapes and ground and low height objects (eg, cars, road furniture etc) are represented by white areas. The second group has the non-ground points which are segmented to extract the planar segments. During segmentation, the building mask is first divided into small grid cells $\left(1 \mathrm{~m}^{2}\right.$ each $)$ and the black cells are clustered based on their locality and number of black pixels. Each cluster indicates an individual building or tree and non-ground points within the cluster are coarsely segmented based on coplanarity and neighbourhood relations. The extracted coarse segments are then refined using a new rule-based procedure where common points among the segments are assigned to the appropriate segments based on their locality and neighbourhood. Finally, another rule-based procedure is followed to remove the planes extracted on trees, which are usually small in size and randomly oriented. The number of unused LIDAR points and the average height difference of the points within a tree segment are high. Experimental results show that the proposed method offers high building detection and roof plane extraction rates when applied to three Australian test areas.

The rest of the paper is organised as follows. Section II presents a comprehensive review of the existing 3D building extraction methods using LIDAR and/or image data. Section III presents the proposed building roof extraction technique. Section IV presents the performance study reported in this study and compares the proposed method with some existing methods. Finally, concluding remarks are presented in Section $\mathrm{V}$.

\section{RELATED WORK}

Although automatic algorithms using raster DSMs (Digital Surface Models) generated from the LIDAR data [3], [4] allow available image processing techniques for faster segmentation, they can suffer from the decreased information in the 3D point cloud. Jochem et al. [5] proposed a roof plane segmentation technique from raster LIDAR data using a seed point based region growing technique. Vegetation was removed using the slope-adaptive LIDAR echo ratio and the approach showed good object-based evaluation results on a large data set using a threshold-free evaluation system.

Among the methods that fully rely on raw LIDAR data, Perera et al. [6] used a surface growing algorithm [7] for segmentation of the point cloud. Then, a cycle graph was used to establish the topological relationship among the line segments extracted along the plane boundaries. This method failed in the absence of missing boundary lines and it displayed low geometric accuracy. Dorninger and Pfeifer [8] proposed a comprehensive method for extraction, reconstruction and regularization of roof planes using LIDAR point clouds. Since the success of the proposed automated procedure was low, the authors advised manual pre-processing and post-processing steps. In the pre-processing step, a coarse selection of building regions was accomplished by digitizing each building interactively. In the post-processing step, the erroneous building models were indicated and rectified by means of commercial 
CAD software. Moreover, some of the algorithmic parameters were set interactively.

Sampath and Shan [9] presented a solution framework for segmentation (detection) and reconstruction of polyhedral building roofs from high density LIDAR data. Similar to the method in [10], the coplanarity of points was determined based on eigenvalue analysis using the Voronoi neighbourhood around each point. The normal vectors were then clustered via the fuzzy k-means algorithm. Good evaluation results for both segmentation and reconstruction were achieved. However, due to the removal of LIDAR points near the plane boundaries, the method exhibited high reconstruction errors for small planes. Furthermore, the fuzzy k-means clustering algorithm is computationally expensive [11]. Kim and Shan [12] also segmented the normal vectors, but they applied a multiphase level set technique.

Tarsha-Kurdi et al. [13] applied an extended robust estimation technique on the regenerated LIDAR point cloud. After converting the original point cloud into a DSM, the missing points were estimated as the mean of the neighbouring points. Then a low-pass filter was applied and the raster point cloud was converted to the raw point cloud. As a result, the regenerated points suffered from decreased positional accuracy. Moreover, the method could not construct planes of less than $50 \mathrm{~m}^{2}$. Sohn et al. [14] clustered building points first based on height similarity and then on planar similarity. They then extracted rectilinear lines and generated polyhedral models from the lines using a binary space partitioning tree. The method produced erroneous results due to improper localisation of the extracted lines or to missing lines. In addition, it failed to separate small roof planes in the clustering algorithm due to use of a predefined bin size for the height histogram. The main problem with the methods using LIDAR data is that the planimetric accuracy is limited by the LIDAR point density.

Methods using ground plans with the LIDAR data [15] simplify the problem by partitioning the given plan and finding the most appropriate planar segments for each partition. Vosselman and Dijkman [15] used the Hough transform to extract planar faces. Schwalbe et al. [16] found the planes as lines after the 2D orthogonal projections of the point clouds using the dominant building orientation. Although the use of ground plan had a positive effect on the reconstruction performance for small buildings usually having flat roofs, decreased the performance for buildings in new-built residential and industrial areas due to erroneous estimation of building orientation. Park et al. [17] followed a region-growing algorithm to extract planar patches initialised by seed clusters having small fitting errors. The technique was able to reconstruct large complex buildings as well as small ones (as small as $4 \mathrm{~m}^{2}$ in area). The major problem with a method using the ground plan is that in the absence of a ground plan, or if the plan is not up to date, the method becomes semi-automatic. In addition, objective evaluation results were missing in many published papers [15], [17].

There are also methods that integrate the image with the LIDAR data usually for improved segmentation results. Rottensteiner et al. [18] improved the geometric quality of the extracted roof plane boundaries from the LIDAR DSM [19] with the help of image lines. Khoshelham et al. [11] applied a split-and-merge technique on a DSM guided image segmentation technique for automatic extraction of roof planes. In evaluation, the accuracy of the reconstructed planes was shown for four simple gable roofs only. Chen et al. [20] reconstructed buildings with straight (flat and gable roofs only) and curvilinear (flat roof only) boundaries from LIDAR and image data. Though the evaluation results were promising, the method could not detect buildings smaller than $30 \mathrm{~m}^{2}$ in area and for the detected buildings both planimetric and height errors were high. Habib et al. [21] reported on semi-automatic polyhedral building model generation through integration of LIDAR data and stereo imagery. Planar roof patches were first generated from the LIDAR data and then 3D image lines were matched along the LIDAR boundaries. Finally, a manual monoplotting procedure was used to both delete incorrect boundaries and add necessary boundary segments. Some true boundaries were missed and erroneous boundaries were detected due to relief displacement, shadows and low image contrast. Rottensteiner [22] segmented multiple aerial images using the classical watershed algorithm and applied the point cloud to determine which image segments corresponded to roof planes. This method required the approximate building boundaries which might be manually set if not available. The approximate floor height of buildings had to be estimated in order to generate the terrain model. However, it may be difficult to estimate the building floor heights in a hilly area where building roofs may have heights similar to the ground. Moreover, the segmentation algorithm suffered from oversegmentation in sunny areas and from under-segmentation in shaded areas. The tuning of segmentation parameters was a real problem. Cheng et al. [23] also integrated multi-view aerial imagery with LIDAR data for 3D building model reconstruction. This was a semi-automatic method since in many cases $20-30 \%$ of roof lines needed to be manually edited. In addition, this method was computationally expensive and failed to reconstruct complex roof structures.

Recently, Awrangjeb et al. [1] used image lines near the non-ground LIDAR points in order to define stable seed regions. A planar segment is initialized using a seed region and then grown iteratively by including neighbouring LIDAR points which are compatible to the plane. Cheng et al. [24] first performed a coarse-to-fine LIDAR segmentation using a robust estimation technique and then applied a shrink-expand technique, where spectral and texture information were used to judge the candidate points, in order to rectify the over- and under-segmentation cases. Lines from the image were used to determine step lines in reconstruction step. Experimental results showed that the method not only missed some small planes but also extracted some fictitious planes. Demir and Baltsavias [25] first detected buildings using a combination of four methods and then extracted planar segments using a robust estimation technique. Finally, 3D lines estimated by plane intersection along with those which were generated by image matching were employed to reconstruct the plane outlines. The method could not extract planes which were close to trees.

\section{Proposed Roof Extraction Method}

Fig. 1 shows an overview of the proposed building roof extraction procedure. The input data consists of raw LIDAR data. In the detection step (top dashed rectangle in Fig. 1), the LIDAR points are classified into two groups: ground points, such as ground, road furniture, cars and bushes that are 


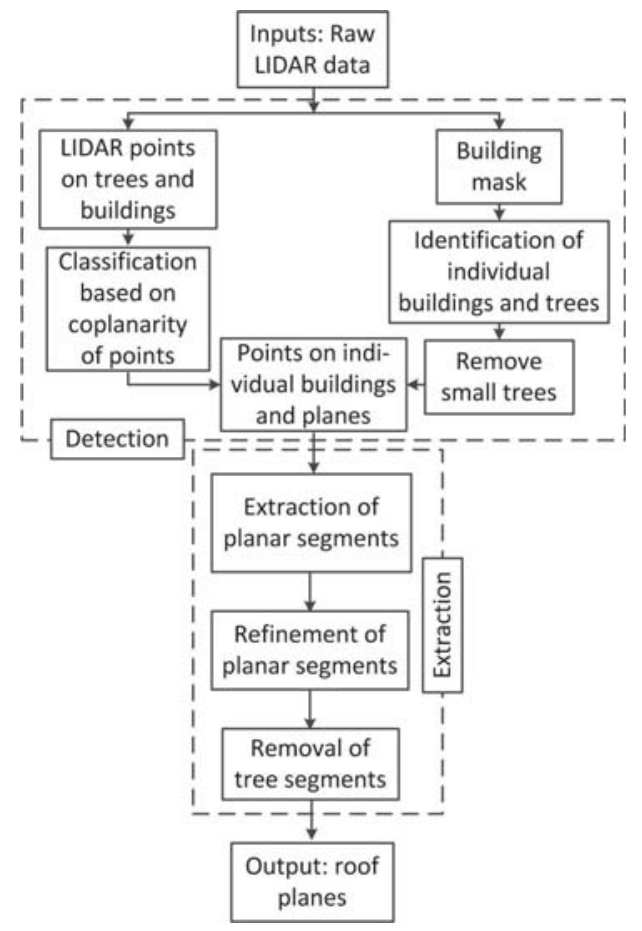

Fig. 1. The proposed roof plane extraction technique.

below the threshold, and non-ground points, which represent elevated objects such as buildings and trees. The building mask, known as the 'ground mask', is generated using the ground points. Individual buildings and trees are obtained as clusters of black pixels in the building mask and trees with low density canopies are removed. The coplanarity of each individual non-ground LIDAR point is decided based on its Delaunay neighbourhood. The planar segments are extracted from the non-ground LIDAR points on individual buildings and trees. The extracted LIDAR segments are then refined using a new rule-based procedure. Finally, the false planes are removed using information such as area and neighbourhood, as well as any point spikes within the planar boundary.

Figs. 2a presents a sample scene from the Aitkenvale data set [1], which will be used to illustrate the different steps of the proposed extraction method. The LIDAR point density in this data set is 29.3 points $/ \mathrm{m}^{2}$.

\section{A. Point Classification and Building Detection}

Using a height threshold, the LIDAR points are classified into two major groups: ground points such as ground, road furniture, cars and bushes that are below the threshold, and non-ground points that represent elevated objects such as buildings and trees. The primary or building mask $M_{g}$, as shown in Fig. 2b, is generated using the ground points following the procedure in [26]. The mask $M_{g}$ indicates the void areas where there are no laser returns below the height threshold, ie, ground areas covered by buildings and trees. Unlike the earlier work [26] that sets a height threshold of $2.5 \mathrm{~m}$, a small threshold of $1 \mathrm{~m}$ has been used in this paper in order to capture the buildings that has similar height as the terrain, specially in the hilly areas.
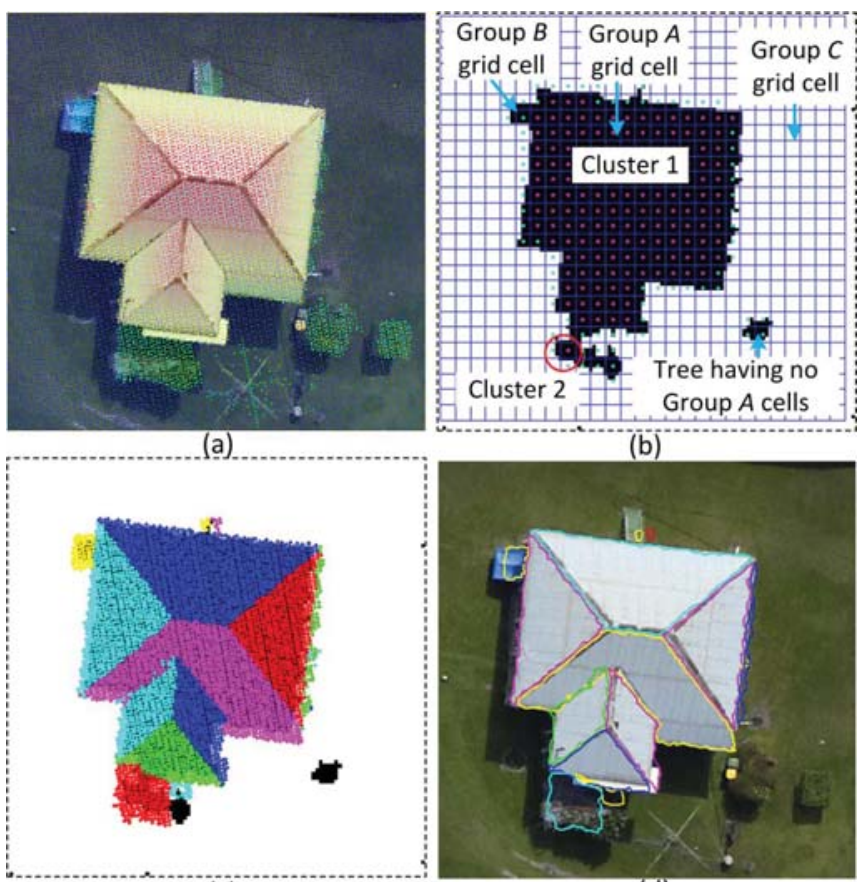

(c)

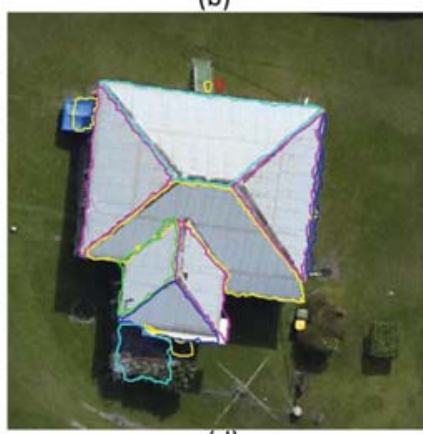

(d)
Fig. 2. A sample scene from the Aitkenvale data set: (a) raw LIDAR points overlaid on an orthoimage, (b) building mask and grouping and clustering of the mask grid cells, (c) extracted planes from Cluster 1 shown in (b), and (d) all plane boundaries from the two clusters shown in (b).

The mask $M_{g}$ is divided into $1 \mathrm{~m}^{2}$ grid cells. Fig. $2 \mathrm{~b}$ shows the grid, whose cells can be categorized into three groups. The cells in Group A, shown in magenta coloured dots in Fig. 2b, contain only black pixels and represent the areas inside the building or tree boundaries. In contrast, the cells in Group $B$ (shown in cyan dots) contain both white and black pixels and represent the areas along the building and tree boundaries. The cells in Group $C$ contain only white pixels and represent the ground. Group $A$ cells are now separated into clusters, where any two Group $A$ cells in each cluster are connected by other Group $A$ cells belonging to the same cluster. As shown in Fig. $2 \mathrm{~b}$, there are 2 such clusters in the sample scene. Thereafter, the Group $B$ cells along the building boundary are added to each of the clusters. Each of the added Group $B$ cells should have at least one Group A cell as its neighbour in the cluster. Finally, for each of the clusters, the cluster boundary is obtained using the Canny edge detector. Lines along the boundary are also extracted following the procedure in [27]. These lines help to locate the roof planes near the building boundary. The nonground LIDAR points within the cluster boundary are assigned to the cluster.

The clustering technique described above helps in the elimination of trees which are not dense (eg, tree at the rightbottom of Fig. 2) and/or small in area. In addition, dense vegetation can be separated into small parts. In such a small vegetated area, it will be impossible to construct a large plane on a small vegetated area. Thus many planes constructed on trees can be easily removed by applying the minimum plane size (see Section III-C). 

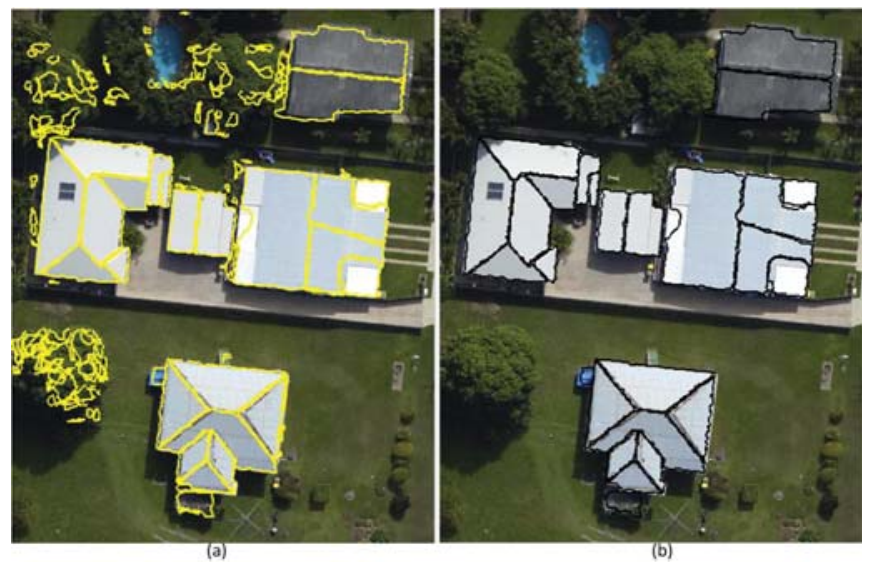

Fig. 3. Removal of false planes: (a) all plane boundaries from the Aitkenvale data set and (b) refined plane boundaries.

\section{B. Extraction of Planar Segments}

By using the Delaunay triangulation algorithm, a natural neighbourhood of non-ground LIDAR points can be generated for either one cluster at a time or all non-ground points at the same time. The neighbourhood of a point $P$ consists of the points $Q_{i}, 1 \leq i \leq n$, where each line $P Q_{i}$ is a side of a Delaunay triangle. The coplanarity of $P$ is decided using its neighbouring points following the procedure in [9]. Points within a roof plane are found to be coplanar and those along the boundary of a plane are generally found to be non-coplanar.

For each cluster, let the two sets of the non-ground LIDAR points be $S_{1}$ containing all the coplanar points and $S_{2}$ containing the rest (non-coplanar). The first planar segment can now be initialised using a coplanar point $P \in S_{1}$. Initially, $P$ can be located along the cluster boundary using the extracted boundary lines. This new planar segment is extended using the neighbouring points from $S_{1}$ and $S_{2}$. Points within a small neighbourhood (within $2 d_{\max }$, where $d_{\max }$ is the maximum point spacing in the data) of the points currently on the plane are tested against the plane equation. If a neighbouring point has a small normal distance to the plane or a similar estimated height using the plane equation, it is included into the current plane. Once its extension is complete, all the coplanar points in the extended planar segment are marked so that none of them is later used for initiating another planar segment. As a result, the points in $S_{2}$, which mainly reside along the plane boundaries, can be used by more than one extracted plane. The second planar segment is grown by using an unused coplanar point (from $S_{1}$ ) which may also be located along the boundary lines. Later, when no coplanar points are found along the boundary lines, an unused coplanar point is randomly selected and a new planar segment is grown. The iterative procedure continues until no coplanar point remains unused.

Fig. 2(c) shows the extracted planar segments and Fig. 2(d) shows their boundaries. Fig. 3a shows all the extracted planar boundaries on the Aitkenvale data set. In order to find the boundary of a plane, the corresponding set of LIDAR points $S_{p}$ is used to generate a binary mask $M_{b}$ (similar to the building mask $M_{g}$ ). The boundary of the plane is the Canny edge around the black shape in $M_{b}$. For each edge point the nearest LIDAR point height from $S_{p}$ is assigned.
TABLE I. DATA SETS (PD = POINT DENSITY IN POINTS $/ \mathrm{M}^{2}, \mathrm{~B}=$ BUILDINGS, B50 = BUILDINGS LARGER THAN 50M ${ }^{2}, \mathrm{P}=$ PLANES, P10 = PLANES LARGER THAN $10 \mathrm{M}^{2}, \mathrm{PR}=$ PROPERTIES, AV $=$ AITKENVALE, EL

= ELTHAM, HT = HOBART, TERRAIN: $\mathrm{F}=$ FLAT AND H = HILLY, VEGETATION: $\mathrm{L}=$ LOW, $\mathrm{M}=$ MODERATE AND $\mathrm{D}=$ DENSE.

\begin{tabular}{l|c|c|c|c|c}
\hline Scenes & Areas & PD & B(B50) & P(P10) & Pr \\
\hline \hline AV & $66 \times 52$ & 29.3 & $5(5)$ & $26(23)$ & F,M \\
\hline EL & $300 \times 300$ & 4.8 & $70(53)$ & $441(343)$ & H,D \\
\hline HT & $222 \times 390$ & 1.6 & $65(43)$ & $257(175)$ & H,D \\
\hline
\end{tabular}

\section{Elimination of Trees}

As shown in Fig. 3a, there are many false planes constructed on trees. In order to remove these false-positive planes, mostly constructed on trees, a new rule-based procedure is proposed. For an extracted LIDAR plane, its area, straight line segments along its boundary, and neighbourhood information, as well as any LIDAR spikes within its boundary, are used to decide whether it is a false alarm. For a given point on the extracted LIDAR plane, the mean height difference with its neighbouring points is also used. This height difference is large for a tree plane, but small for a roof plane. The average height difference for a plane is estimated from individual height differences within the plane. A LIDAR plane fitted on a tree is usually small in size and there may be some LIDAR spikes within its boundary. Moreover, there may be a large number of unused (ie, not on any of the extracted planes) LIDAR points within the boundary of a tree plane. The number of points used by the extracted planes is usually low on a tree cluster, but high on a building cluster. Moreover, there may be some long straight line segments (at least $3 \mathrm{~m}$ long) along the boundary of a roof plane.

Fig. $3 b$ shows the refined plane boundaries for the Aitkenvale data set. An individual building can now be easily obtained as a group of planes. All the LIDAR points from the group of planes are used together to extract the corresponding building boundary. A binary mask is formed using these points and the boundary of the building is the Canny edge around the black shape in the mask. The height of the nearest LIDAR point of the building is assigned to each edge point.

\section{RESULTS AND DISCUSSIONS}

Table I shows the characteristics of the three Australian data sets employed in this study. While the AV data set has moderate vegetation on a flat terrain, the EL and HT data sets have dense vegetation on hilly areas. Many of the buildings are severely occluded by the surrounding trees. Two dimensional reference data sets were created by monoscopic image measurement using the Barista software [28]. All visible roof planes were digitized as polygons irrespective of their size. The reference data therefore included garden sheds, garages, etc. that were sometimes as small as $1 \mathrm{~m}^{2}$ in area.

The objective evaluation followed a previously proposed automatic and threshold-free evaluation system [29], [30]. It establishes pseudo one-to-one correspondences between the reference and the detected entity sets. It uses a point-inpolygon approach to decide whether a detected entity overlaps a reference entity. The system considers object-based evaluation where the number of true positives, false positives and false negatives is used. In addition, the system also uses the geometric evaluation where the root mean square error 
TABLE II. OBJECT-BASED AND GEOMETRIC EVALUATION RESULTS FOR BUILDING DETECTION: $C_{m}=$ COMPLETENESS, $C_{r}=$ CORRECTNESS AND $Q_{l}=$ QUALITY $\left(C_{m}, 50, C_{r, 50}\right.$ AND $Q_{l, 50}$ ARE FOR BUILDINGS OVER $50 \mathrm{M}^{2}$ ) ARE IN PERCENTAGE; $R M S E=$ PLANIMETRIC ACCURACY IN METRE.

\begin{tabular}{l||c|c|c|c|c|c|c}
\hline Areas & $C_{m}$ & $C_{r}$ & $Q_{l}$ & $C_{m, 50}$ & $C_{r, 50}$ & $Q_{l, 50}$ & $R M S E$ \\
\hline \hline AV & 100 & 100 & 100 & 100 & 100 & 100 & 0.44 \\
\hline EL & 77.6 & 88.2 & 70.3 & 100 & 88 & 88 & 1.31 \\
\hline HT & 71.2 & 80.8 & 60.9 & 100 & 80.8 & 80.8 & 1.33 \\
\hline
\end{tabular}

(RMSE) between two corresponding detected and reference entities is used for planimetric (2D) accuracy and the average height error between the actual LIDAR heights and estimated heights of all the points on an extracted plane is used as height accuracy. Three metrics are used for object-based evaluation in both roof and plane levels: completeness, correctness and quality. The formal definitions and formulas of these metrics can be found in [29], [26].

The results and discussions on the test data sets are presented below for building detection and roof plane extraction separately.

\section{A. Building detection results}

Table II shows object-based and geometric evaluation results for building detection. Fig. 4 shows the detected buildings for the Hobart data set.

In object-based evaluation (Table II), the proposed method performed the best in the AV data set. The reason is that this scene is flat in nature and has moderate vegetation. Furthermore, buildings in this area are not occluded at all. In contrast, the EL and HT data sets are more complex having lots of vegetation on hilly areas. Compared to the HT data set, the EL is more hilly and has more occluded buildings. However, the proposed algorithm performed better in the EL data set than in the HT data set. It is because point density in the EL data set is higher than that in the HT data set. In both data sets, some large trees could not be removed, as indicated within yellow coloured ellipses in Figs. 4 and 5. As a result, the correctness values are less than $90 \%$ even for buildings larger than $50 \mathrm{~m}^{2}$ in area. Some of the detected trees in the EL data set were highly dense, so laser points hardly reflected from the ground, trunks or other parts of the trees. Some of the detected tree tops in the HT data set were not only highly dense, but also shaped such that they seemed to be flat planes. Due to the above phenomena, the extracted planes on these trees were large and could not be removed using the techniques presented in Section III-C. Moreover, many of the garden sheds and car ports could not be detected due to occlusion in these two areas (see examples in Figs. 4(d)-(e)).

The accuracy of the extracted building boundaries is one to two times of the maximum point spacing in the input LIDAR data. While for the AV data set the RMSE value is $0.44 \mathrm{~m}$, for the EL and HT data set it is more than $1.2 \mathrm{~m}$. It shows that better planimetric accuracy is possible with high density LIDAR data.

\section{B. Roof plane extraction results}

Tables III shows object-based and geometric evaluation results for roof plane extraction. Fig. 5 shows the extracted
TABLE III. OBJECT-BASED AND GEOMETRIC EVALUATION RESULTS FOR ROOF PLANE EXTRACTION: $C_{m}=$ COMPLETENESS, $C_{r}=$

CORRECTNESS AND $Q_{l}=$ QUALITY $\left(C_{m, 10}, C_{r, 10}, Q_{l, 10}\right.$ ARE FOR BUILDINGS OVER $\left.10 \mathrm{M}^{2}\right) ; R M S E=$ PLANIMETRIC AND $R M S Z=$ HEIGHT ACCURACIES IN METRE.

\begin{tabular}{l||c|c|c|c|c|c|c|c}
\hline Areas & $C_{m}$ & $C_{r}$ & $Q_{l}$ & $C_{m, 10}$ & $C_{r, 10}$ & $Q_{l, 10}$ & $R M S E$ & $R M S Z$ \\
\hline \hline AV & 92.3 & 92.3 & 85.7 & 96 & 92.3 & 88.9 & 0.49 & 0.032 \\
\hline EL & 79.1 & 87.9 & 71.4 & 92.6 & 87.9 & 82.1 & 0.88 & 0.037 \\
\hline HT & 69.4 & 69.6 & 53.3 & 91 & 69.6 & 65.2 & 1.00 & 0.042 \\
\hline
\end{tabular}

planes for the Eltham data set. ${ }^{1}$

In object-based evaluation (Table III), the completeness values are smaller than the correctness values for almost all data sets. This phenomenon indicates that the method either could not extract some of the small roof planes or merged them with the neighbouring large planes. For instance, Fig. 5(b)(c) show some missing planes, marked in yellow squares. For planes larger than $10 \mathrm{~m}^{2}$, the completeness values are higher than the correctness values in all data sets. This is because in these data sets many of the extracted roof planes on trees were larger than $10 \mathrm{~m}^{2}$, as shown within ellipses in Fig. 5(a).

Similar to building detection results discussed above, the proposed method performed better in the AV data set. For roof planes larger than $10 \mathrm{~m}^{2}$, it extracted more than $95 \%$ of the planes completely in this data set.

In terms of planimetric accuracy, the proposed method again offers 1 to 2 times of the point spacing in the input data. The height error is only 3 to $4 \mathrm{~cm}$, which shows the advantage of using raw LIDAR data, in stead of raster LIDAR data or stereo data.

\section{Comparisons}

A recent method by Awrangjeb et al. [1] showed both object- and pixel-based evaluation results on the AV data set which has been used in the current study. The previous method performed similar to the current method in this data set. However, in other test data sets, the previous method failed to extract the corresponding small roof planes due to absence of image lines. It also could not extract some small buildings like garden sheds and car ports. In contrast, the proposed method is capable of detecting buildings smaller than $10 \mathrm{~m}^{2}$ and the roof planes as small as $1 \mathrm{~m}^{2}$ in area. Furthermore, the proposed method works only on the LIDAR data, so it offers a flexibility in application where the orthoimage is not available.

All three data sets have previously been employed by a building detection technique presented in Awrangjeb et al. [31] (see more results in [32], [33]). This technique is capable of detecting buildings in complex scenes with high vegetation and hilly areas. However, it has the following shortcomings. Firstly, each of the detected building boundaries is approximated by one or more rectangles. However, a complex building roof may not be well approximated using one or two rectangles only. Second, it fails to detect buildings smaller than $10 \mathrm{~m}^{2}$. The proposed approach in the current submission not only offers a better approximation of a complex building boundary by a polygonal representation, but also is capable of detecting small buildings. As a result, it performed better in all data sets in

\footnotetext{
${ }^{1}$ All results and data sets are available at http://users.monash.edu.au/
} $\sim$ mawrangj/RExtraction.html. 


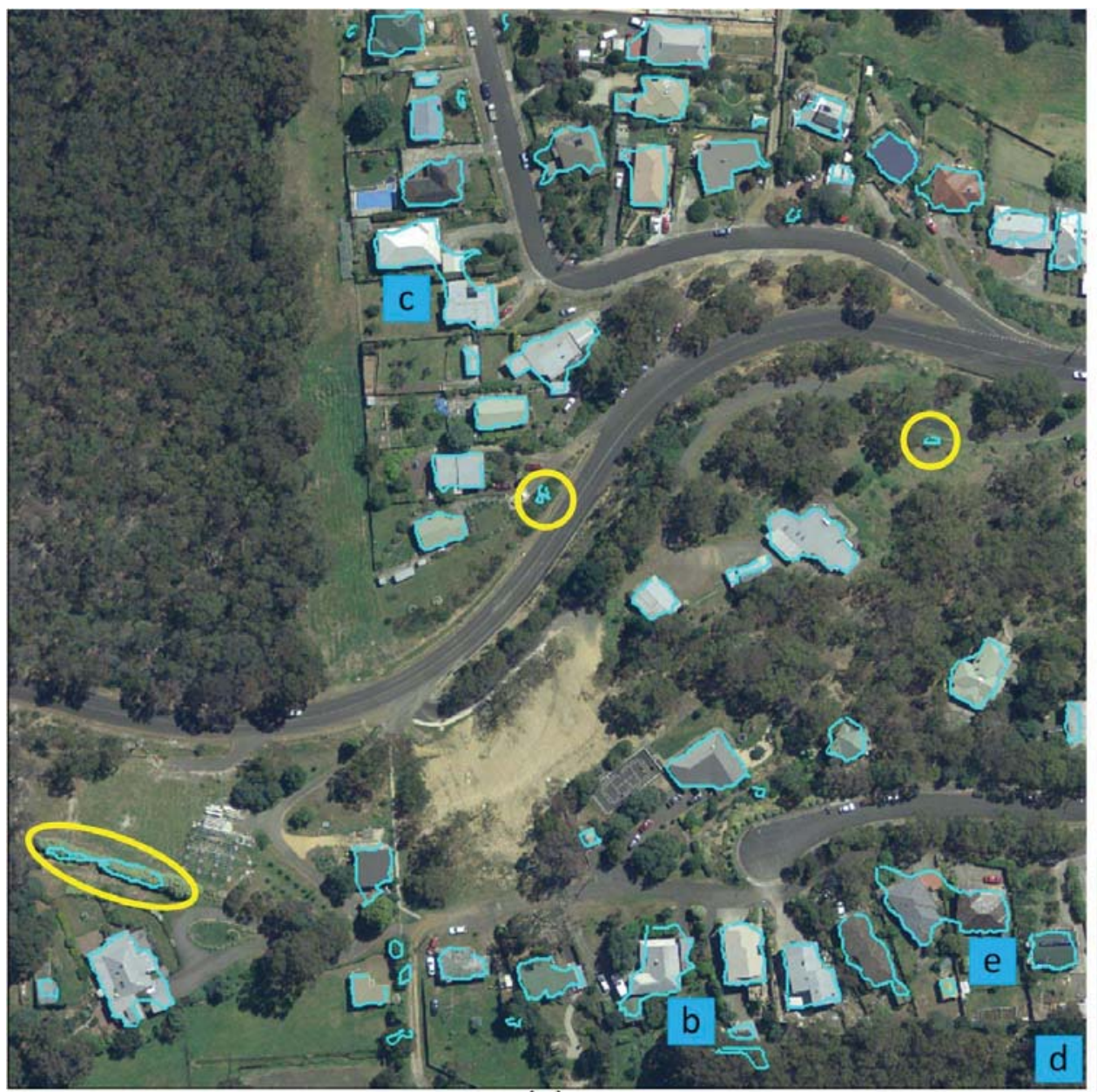

(a)

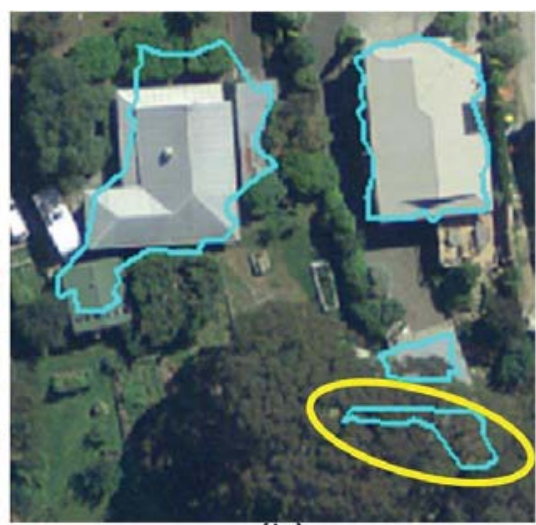

(b)

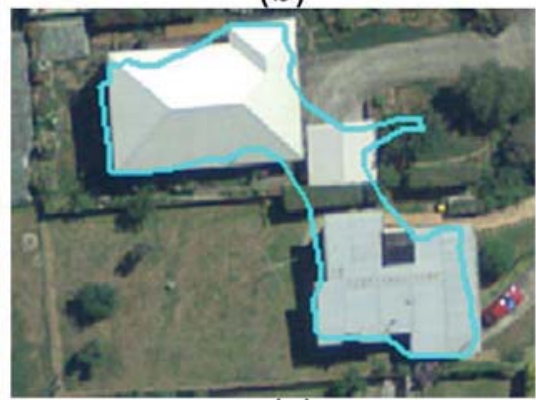

(c)

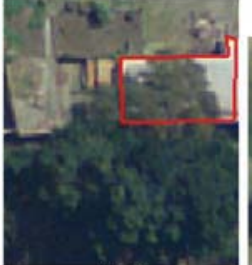

(d)

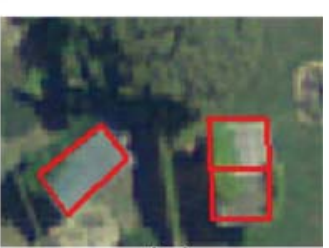

(e)

Fig. 4. Building detection on (a) Hobart data set, (b)-(c) some detection examples on complex cases, and (d)-(e) some missing buildings in difficult cases (small in size and/or occluded by trees). Areas marked by letters in (a) are magnified in (b) to (e).

object- and pixel-based evaluations as well as in the geometric accuracy.

\section{CONCLUSION}

A new LIDAR point cloud segmentation algorithm has been proposed for automatic extraction of 3D building roof planes from the raw LIDAR data. Firstly, the raw LIDAR data is divided into ground and non-ground points and a building mask is generated from the ground points. The black areas in the building mask represent the covered areas (ie, buildings and trees) on the ground where no laser returns below a certain height. The building mask is divided into $1 \mathrm{~m}^{2}$ grid cells and the black cells are clustered such that each cluster represents an individual building or tree. The non-ground points within a cluster are then coarsely segmented based on their coplanarity and neighbourhood relations. The planar segments are refined using a rule-based procedure where common points between planar segments are assigned to the appropriate planes based on the relationship between the neighbouring planes and the locality of the points. Finally, another rule-based procedure is applied to remove tree planes which are small in size and randomly oriented. Within the area of an extracted plane on a tree, the average LIDAR height difference and the number of unused (not used for plane estimation) LIDAR points are usually high.

It has been shown via experimental testing that the proposed algorithm affords high building detection and roof plane extraction performance. It is not only capable of detecting small buildings but can also extract small roof planes on complex building roofs. Moreover, in most cases it can separate buildings which are surrounded by dense vegetation.

\section{ACKNOWLEDGMENT}

Dr. Awrangjeb is a recipient of the Discovery Early Career Researcher Award by the Australian Research Council (project number DE120101778). The authors would like to thank Ergon Energy (www.ergon.com.au) for providing the Aitkenvale data set, Department of Sustainability and Environment (www.dse.vic.gov.au) for the Eltham data set and Photomapping services (www.photomapping.com.au) for the Hobart data set. 


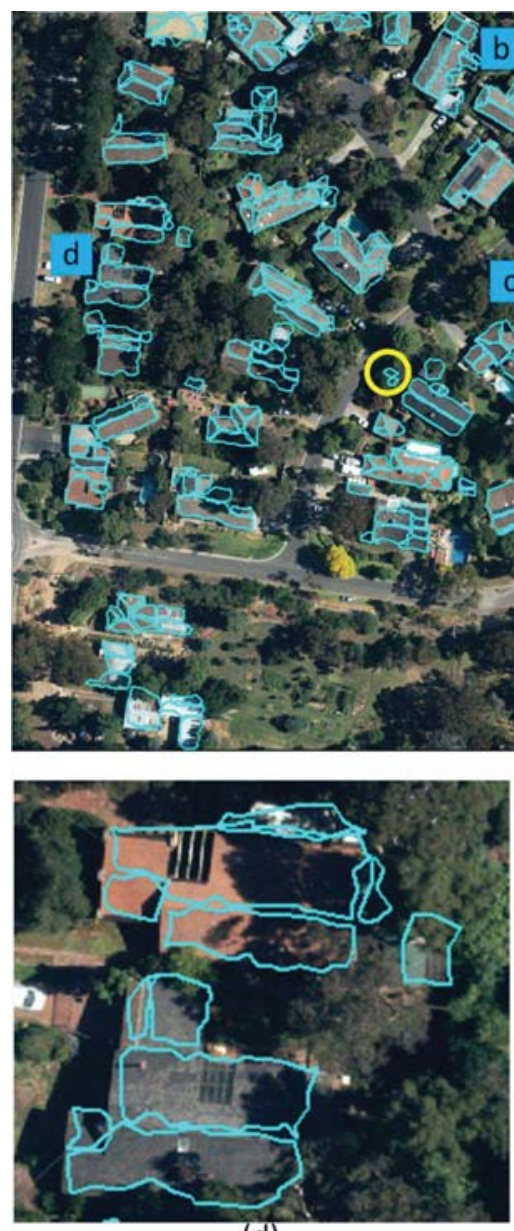

(d)

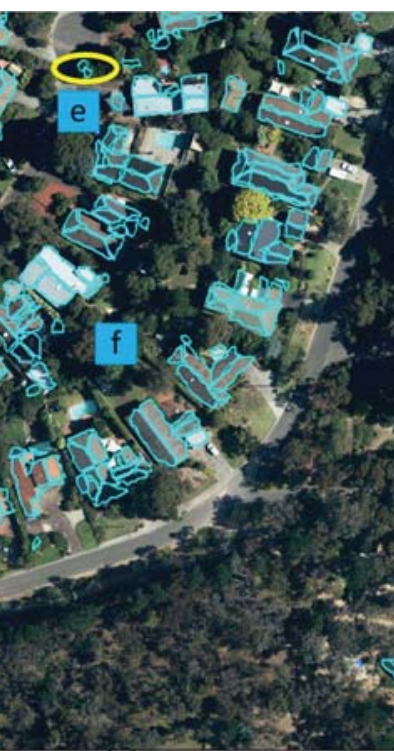

(a)

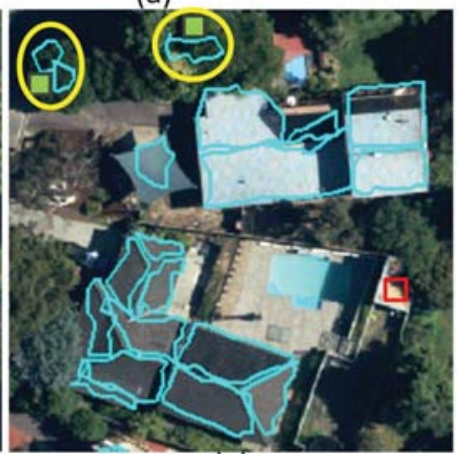

(e)
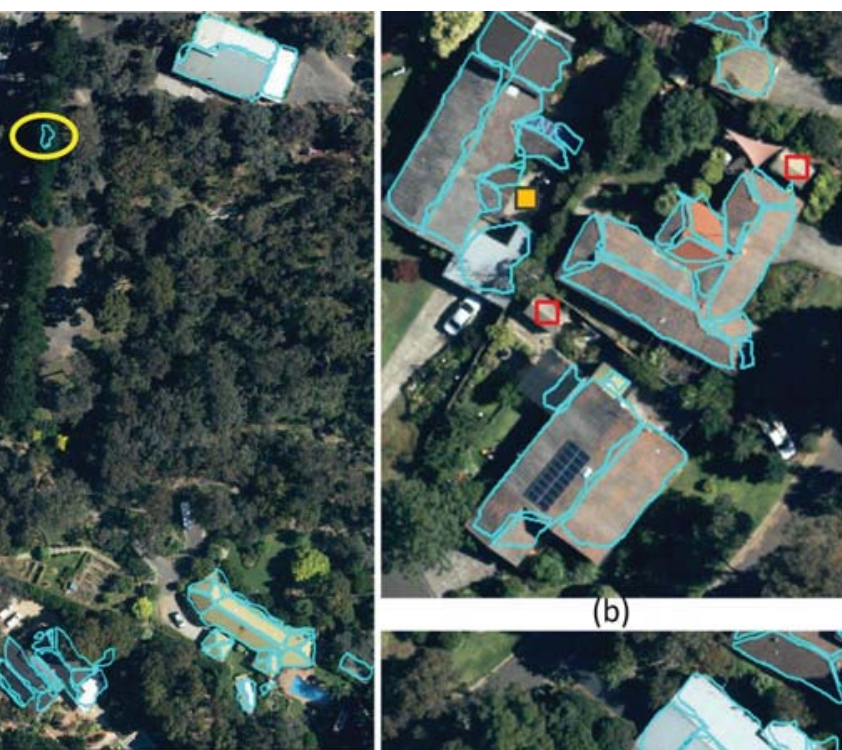

(b)

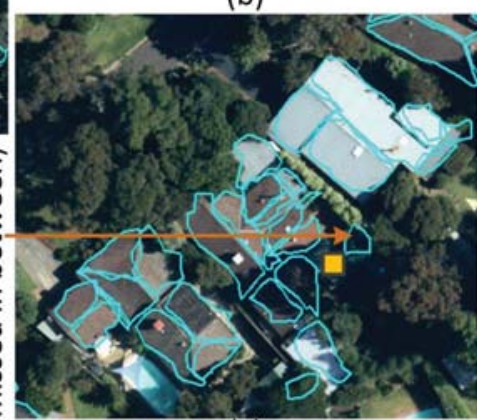

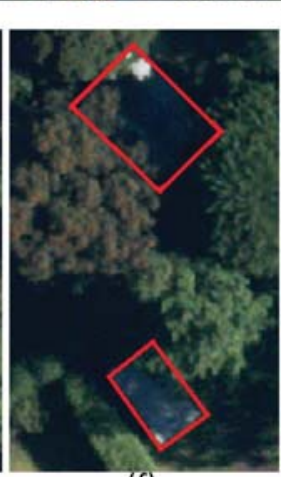

(f) (c)

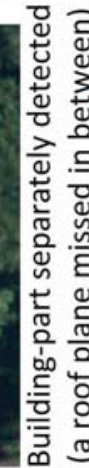

ก 0 extracted planes

bे

은 은 $\square$ extracted false (trees) planes T

Fig. 5. Roof extraction on (a) Eltham data set, (b)-(e) some extracted planes on complex cases, and (f) some missing planes (buildings) in difficult cases. Areas marked by letters in (a) are magnified in (b) to (f).

\section{REFERENCES}

[1] M. Awrangjeb, C. Zhang, and C. S. Fraser, "Automatic extraction of building roofs using lidar data and multispectral imagery," ISPRS Journal of Photogrammetry and Remote Sensing, vol. 83, no. 9, pp. 1-18, 2013.

[2] N. Haala and M. Kada, "An update on automatic 3d building reconstruction," ISPRS Journal of Photogrammetry and Remote Sensing, vol. 65, no. 6, pp. 570-580, 2010.

[3] F. Rottensteiner and C. Briese, "Automatic generation of building models from lidar data and the integration of aerial images," in Proc. ISPRS working group III/3 workshop on 3-D reconstruction from airborne laserscanner and InSAR data, vol. IAPRS XXXIV, Part 3/W13, Dresden, Germany, 2003, pp. 512-517.

[4] D. Chen, L. Zhang, J. Li, and R. Liu, "Urban building roof segmentation from airborne lidar point clouds," International Journal of Remote Sensing, vol. 33, no. 20, pp. 6497-6515, 2012.

[5] A. Jochem, B. Hfle, V. Wichmann, M. Rutzinger, and A. Zipf, "Areawide roof plane segmentation in airborne lidar point clouds," Computers, Environment and Urban Systems, vol. 36, no. 1, pp. 54-64, 2012.

[6] S. N. Perera, H. A. Nalani, and H.-G. Maas, "An automated method for $3 \mathrm{~d}$ roof outline generation and regularization in airborne laser scanner data," in ISPRS Annals of the Photogrammetry, Remote Sensing and Spatial Information Sciences, vol. I-3, Melbourne, Australia, 2012, pp. 281-286.

[7] G. Vosselman, B. G. H. Gorte, G. Sithole, and T. Rabbani, "Recognising structure in laser scanner point cloud," in International Archives of
Photogrammetry, Remote Sensing and Spatial Information Sciences, vol. XXXVI (8/W2), istanbul, Turkey, 2004, pp. 33-38.

[8] P. Dorninger and N. Pfeifer, "A comprehensive automated 3d approach for building extraction, reconstruction, and regularization from airborne laser scanning point clouds," Sensors, vol. 8, no. 11, pp. 7323-7343, 2008.

[9] A. Sampath and J. Shan, "Segmentation and reconstruction of polyhedral building roofs from aerial lidar point clouds," IEEE Transactions on Geoscience and Remote Sensing, vol. 48, no. 3, pp. 1554-1567, 2010.

[10] V. Verma, R. Kumar, and S. Hsu, "3d building detection and modeling from aerial lidar data," in IEEE Computer Society Conference on Computer Vision and Pattern Recognition, vol. 2, New York, USA, 2006, pp. 2213-2220.

[11] K. Khoshelham, Z. Li, and B. King, "A split-and-merge technique for automated reconstruction of roof planes," Photogrammetric Engineering \& Remote Sensing, vol. 71, no. 7, pp. 855-862, 2005.

[12] K. Kim and J. Shan, "Building roof modeling from airborne laser scanning data based on level set approach," ISPRS Journal of Photogrammetry and Remote Sensing, vol. 66, no. 4, pp. 484-497, 2011.

[13] F. Tarsha-Kurdi, T. Landes, and P. Grussenmeyer, "Extended RANSAC algorithm for automatic detection of building roof planes from lidar data," The photogrammetric journal of Finland, vol. 21, no. 1, pp. 97109, 2008.

[14] G. Sohn, X. Huang, and V. Tao, "Using binary space partitioning tree for reconstructing polyhedral building models from airborne lidar data," 
Photogrammetric Engineering \& Remote Sensing, vol. 74, no. 11, pp. 1425-1438, 2008.

[15] G. Vosselman and S. Dijkman, "3d building model reconstruction from point clouds and ground plans," International Archives of the Photogrammetry and Remote Sensing, vol. XXXIV, no. 3/W4, pp. 3744, 2001.

[16] E. Schwalbe, H. G. Maas, and F. Seidel, "3d building model generation from airborne laser scanner data using $2 \mathrm{~d}$ gis data and orthogonal point cloud projections," in Proceedings of the ISPRS Workshop Laser scanning 2005. ISPRS Archives, vol. 36(3), Enschede, The Netherlands, 2005, pp. 209-214.

[17] J. Park, I. Y. Lee, Y. Choi, and Y. J. Lee, "Automatic extraction of large complex buildings using lidar data and digital maps," International Archives of the Photogrammetry and Remote Sensing, vol. XXXVI, no. 3/W4, pp. 148-154, 2006.

[18] F. Rottensteiner, J. Trinder, S. Clode, and K. Kubik, "Fusing airborne laser scanner data and aerial imagery for the automatic extraction of buildings in densely built-up areas," in Proc. ISPRS Twentieth Annual Congress, Istanbul, Turkey, 2004, pp. 512-517.

[19] F. Rottensteiner, "Automatic generation of high-quality building models from lidar data," Computer Graphics and Applications, vol. 23, no. 6, pp. 42-50, 2003.

[20] L. Chen, T. Teo, C. Hsieh, and J. Rau, "Reconstruction of building models with curvilinear boundaries from laser scanner and aerial imagery," Lecture Notes in Computer Science, vol. 4319, pp. 24-33, 2006.

[21] A. F. Habib, R. Zhai, and K. Changjae, "Generation of complex polyhedral building models by integrating stereo-aerial imagery and lidar data," Photogrammetric engineering \& remote sensing, vol. 76, no. 5, pp. 609-623, 2010.

[22] F. Rottensteiner, "Roof plane segmentation by combining multiple images and point clouds," International Archives of Photogrammetry, Remote Sensing and Spatial Information Sciences, vol. 38, no. 3A, pp. 245-250, 2010

[23] L. Cheng, J. Gong, M. Li, and Y. Liu, " $3 \mathrm{~d}$ building model reconstruction from multi-view aerial imagery and lidar data," Photogrammetric Engineering \& Remote Sensing, vol. 77, no. 2, pp. 125-139, 2011.

[24] L. Cheng, L. Tong, Y. Chen, W. Zhang, J. Shan, Y. Liu, and M. Li, "Integration of lidar data and optical multi-view images for $3 \mathrm{~d}$ reconstruction of building roofs," Optics and Lasers in Engineering, vol. 51, no. 4, pp. 493-502, 2013.

[25] N. Demir and E. Baltsavias, "Automated modelling of 3d building roofs using image and lidar data," in ISPRS Annals of the Photogrammetry, Remote Sensing and Spatial Information Sciences, vol. I-4, Melbourne, Australia, 2012, pp. 35-40.

[26] M. Awrangjeb, M. Ravanbakhsh, and C. S. Fraser, "Automatic detection of residential buildings using lidar data and multispectral imagery," ISPRS Journal of Photogrammetry and Remote Sensing, vol. 65, no. 5, pp. 457-467, 2010.

[27] M. Awrangjeb and G. Lu, "Robust image corner detection based on the chord-to-point distance accumulation technique," IEEE Transactions on Multimedia, vol. 10, no. 6, pp. 1059-1072, Oct 2008.

[28] Barista. (2011) The barista software. [Online]. Available: www. baristasoftware.com.au

[29] M. Awrangjeb, M. Ravanbakhsh, and C. S. Fraser, "Building detection from multispectral imagery and lidar data employing a thresholdfree evaluation system," International Archives of the Photogrammetry, Remote Sensing and Spatial Information Sciences, vol. 38, no. part 3A, pp. 49-55, 2010.

[30] M. Awrangjeb and C. S. Fraser, "Automatic and threshold-free evaluation of $3 \mathrm{~d}$ building roof recosntruction techniques," in IEEE International Geoscience and Remote Sensing Symposium, Melbourne, Australia, 2013, pp. 3970-3973.

[31] M. Awrangjeb, C. Zhang, and C. S. Fraser, "Building detection in complex scenes thorough effective separation of buildings from trees," Photogrammetric Engineering \& Remote Sensing, vol. 78, no. 7, pp. 729-745, 2012.

[32] _ "An improved building detection technique for complex scenes," in IEEE International Conference on Multimedia and Expo Workshops, Melbourne, Australia, 2012, pp. 516-521.
[33] M. Awrangjeb and C. S. Fraser, "Effective building detection on complex scenes," in IEEE International Geoscience and Remote Sensing Symposium, Melbourne, Australia, 2013, pp. 1533-1536. 